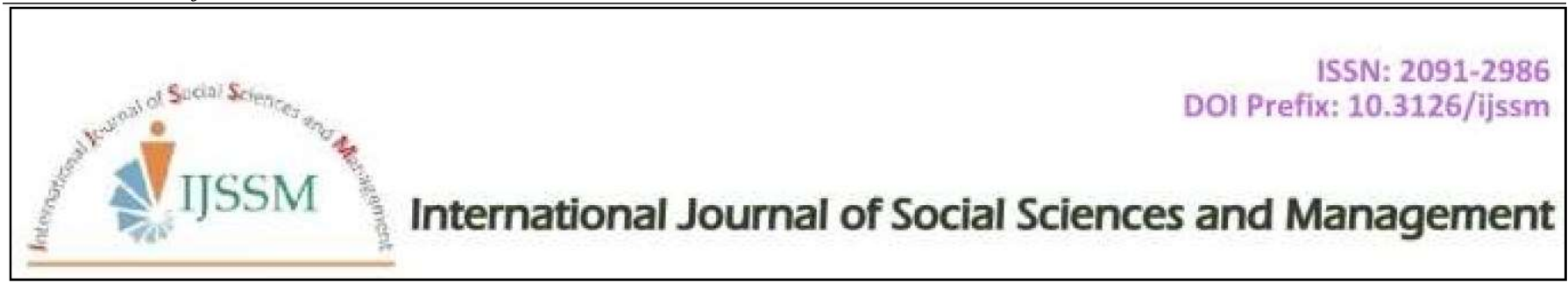

Research Article

\title{
Social and Economic Implications of Old Age Allowance in Nepal: A Study of Kathmandu District (Ward Number 33)
}

\author{
Anupama Dhakal ${ }^{1}$, Utsab Bhattarai ${ }^{2 *}$ \\ ${ }^{1}$ Padma Kanya Multiple Campus, Tribhuvan University, Kathmandu, Nepal \\ ${ }^{2}$ Institute for Culture and Society, Western Sydney University, Locked bag 1797 Penrith, NSW 2751, Australia
}

\begin{abstract}
Article Information
Received: 19 September 2020

Revised version received: 13 October 2020

Accepted: 16 October 2020

Published: 29 October 2020

Cite this article as:

A. Dhakal and U. Bhattarai (2020) Int. J. Soc. Sc.

Manage. 7(4): 248-255.

DOI: $\underline{10.3126 / \text { ijssm.v7i4.32476 }}$

*Corresponding author

Utsab Bhattarai,

Institute for Culture and Society, Western Sydney University, Locked bag 1797 Penrith, NSW 2751, Australia

Email: utsabbhattarai61@gmail.com

Peer reviewed under authority of IJSSM

(C) 2020 IJSSM, Permits unrestricted use under the CC-By-NC license.
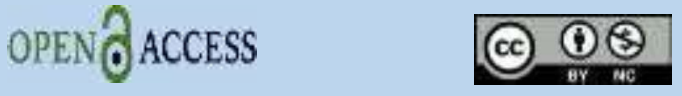

This is an open access article \& it is licensed under a Creative Commons Attribution Non-Commercial 4.0 $\underline{\text { International }}$

(https://creativecommons.org/licenses/by-nc/4.0/)

Keywords: elderly; allowance; social; security; economic
\end{abstract}

\section{Introduction}

Old people (worldwide usage) are often known as seniors (American usage), senior citizens (British and American usage), older adults (in the social sciences), elders (in many cultures including the cultures of aboriginal people), elderly and so on (Jadhav, 2018). Since the meaning of old age varies culturally

\begin{abstract}
Our assumption was that the money disbursed in the form of Old Age Allowance (OAA) in Nepal should have helped in lessening at least some of the financial burdens of its recipients while also enriching their social status and honor as senior citizens. With this assumption and the limitations of time and money, Ward Number 33 of Kathmandu District was selected as our study site. The objective of this study to explore and identify key implications of OAA specifically on the aspects of social and economic life of elderlies. The findings of this study are the outcomes of the analysis of data obtained through fifty face to face household surveys and direct observation of the first author during the fieldwork of five months in 2013. The study results revealed moderate implications of OAA on economic as well as social life of elderlies, however with greater insinuations for the earlier facet compared to later. Participants deemed a significant increment in the amount of OAA for its better outcomes. The value of OAA in 2013 was Nepalese Rupees (Nrs.) 500 which was considered very nominal by all participants. Most of the participants argued that the senior citizens in Nepal should also receive decent discounts/concessions at least on all modes of public transportation as well as health facilities. We strongly recommend that the ministries of Nepal, chiefly, Finance; Women, Children and Senior Citizens; and Federal Affairs and General Administration, including their line agencies must undertake timely reviews and rigorous evaluations of the effectiveness of OAA in compliance with the principles of social security system to ensure social security benefits of the senior citizens of the country.
\end{abstract}

and historically, it is sometimes perceived as "a social construct" rather than a definite "biological stage" (Cossar, 2012). The World Health Organization (2010) mentions that 65 years is the standard age for a person to be 'elderly' or older based on the instances from many developed countries. However, there is no United Nations standard numerical criterion for the definition of elderly (Agrawal, 
2016). In the context of Nepal, Senior Citizens Act 2063 defines a person aged 60 and above as senior citizen (Khanal, 2013). However, the age bar for elderlies varies in different service sectors of Nepal. For example, while the retirement age for military services for lower class is 45 to 48 years, it is 58 years for general government service, and likewise 63 years in the case of university teachers and judiciary services (Geriatric Center Nepal, 2010). Generally, the term 'elderly' is used to refer those individuals who are at their later years and thus unable to support themselves mostly due to bodily ailments associated with old age, which is often considered 60 and virtually over that (Nyanguru, 2007).

In Nepal, there were 2.1 million elderly people representing $8.14 \%$ of the total population of the country in 2011 (Central Bureau of Statistics [CBS], 2011). Improved life span has increased the number of elderly population in Nepal (Ghimire et al., 2018). During 1991-2001, the annual elderly population growth rate was $3.39 \%$, higher than the annual population growth rate of $2.3 \%$ (Pun et al., 2009). Hence, the Government of Nepal has been attempting to implement various population control programs. While the outcomes of such programmes have helped to lower birth rate, proportion of the elderly population is still higher (Chalise, 2006). This is not only the case of Nepal but also of many countries all over the globe (Mosher, 2017). Moreover, the elderlies of Nepal are observed as one of the most neglected social groups within the country (Parker et al., 2014). It is reported that acute economic pressures, tendencies of disintegrating from joint family system and mobility are some of the key obstacles to hider cultural and traditional value systems of many communities in Nepal (Regmi, 1993). Governmental provisions of taking care of its elderly citizens in Nepal is also rare and limited (Khanal, 2013). South Asian Network of Economic Institutes (SANEI) shares the fact about a need of recognition to look after their elderly population by the government, private sector and civil society (Shrestha \& Satyal, 2003). In addition, the political parties of Nepal are also viewed to have influential roles in this regard.

The problem of political instability, poverty, and unemployment compels many youths and adults of Nepal to flee abroad every year in search of study or employment opportunities. This might be one of the reasons why a significant number of aged populations are seen in the country. Elderlies are usually considered as a group of inactive population who face lots of problems (Donaldson, et al., 2015). However, the tradition of providing help by elderlies in a family to their other members is a common practice among all societies in the world. In Nepal, most of the common help that elderlies provide to their families are with kitchen works such as; cooking, chopping vegetables, sweeping, washing, and taking care of grandchildren, livestock, gardening, depending on the age and gender factors of the elderlies. Several studies have been conducted in the past to learn, explore, and analyze the situations of elderly people in Nepal. However, these studies have focused on revealing macro level impacts of OAA. For example, Nepal Participatory Action Network (NEPAN) under the assistance of HelpAge International conducted a field research in 2003 to explore socioeconomic conditions of the elderly people of Nepal. A study conducted by (Pun et al., 2009) analyzed social change among the senior citizens of Nepal, especially among the selected caste and ethnic groups. Likewise, a study was conducted to analyze comprehensive profile of Social Security/ Social Protection program of Nepal (Khanal, 2013). This assessment aimed at evaluating the outcomes and identifying existing problems in the social security program of Nepal both in its formal as well as informal economies emphasizing to workers and families in the informal economy. After an extensive review of the relevant literature, we found a lack of studies in exploring the implications of OAA at micro level, such as lower administrative units of Nepal, which is why this study has been proposed. Concerning to the issues of elderly people in Nepal, several legal provisions have been endorsed by different governments over different periods of time in the country. Nepal's Civil Code 1963 states that the caretaker of parents (whether son or daughter would take care of them at their old age) needs to be clearly mentioned in the Bandapatra. Local Self Governance Act 1999 is another important legislation that makes decisions on matters affecting the lives of the peoples at lower administrative units including protection and development of orphan children, helpless, women, older people and disabled. Similarly, Senior Citizen Policy 2058 is a key policy document of Nepal concerned with the issues of its elderly citizens based on the working plan as guided by Vienna Conference and the United Nation Principles for Ageing (Geriatric Center Nepal, 2010). National Plan of Action on Ageing 2062 is also another document that identifies elderly citizens as one of its main target groups based on the principle that suggests institutional support to be the last resort. Likewise, Senior Citizen Act- 2063 and Regulations- 2065 ensures the social, economic and human rights of the elderly citizens. The purpose of this Act is to protect and provide social security of old age citizens of the country. The Ninth Five-year Plan (1997-2002) of Nepal marked the policy platform to improve livelihood situations of elderly people with emphasis on actions that would reinforce their self-respect, economic opportunities, respect and social security (Geriatric Center Nepal, 2010).

\section{Materials and Methods}

The study site of this research is Ward Number 33 of Kathmandu District which is enclosed by the Dhobi Khola (River) to its east and Ward Number 1 and 31 to the west, Ward Number 5 to the north and Ward Number 32 to the south (see Fig 1). It is situated at the heart of the district covering an affluent area of 85.7 hectares. 


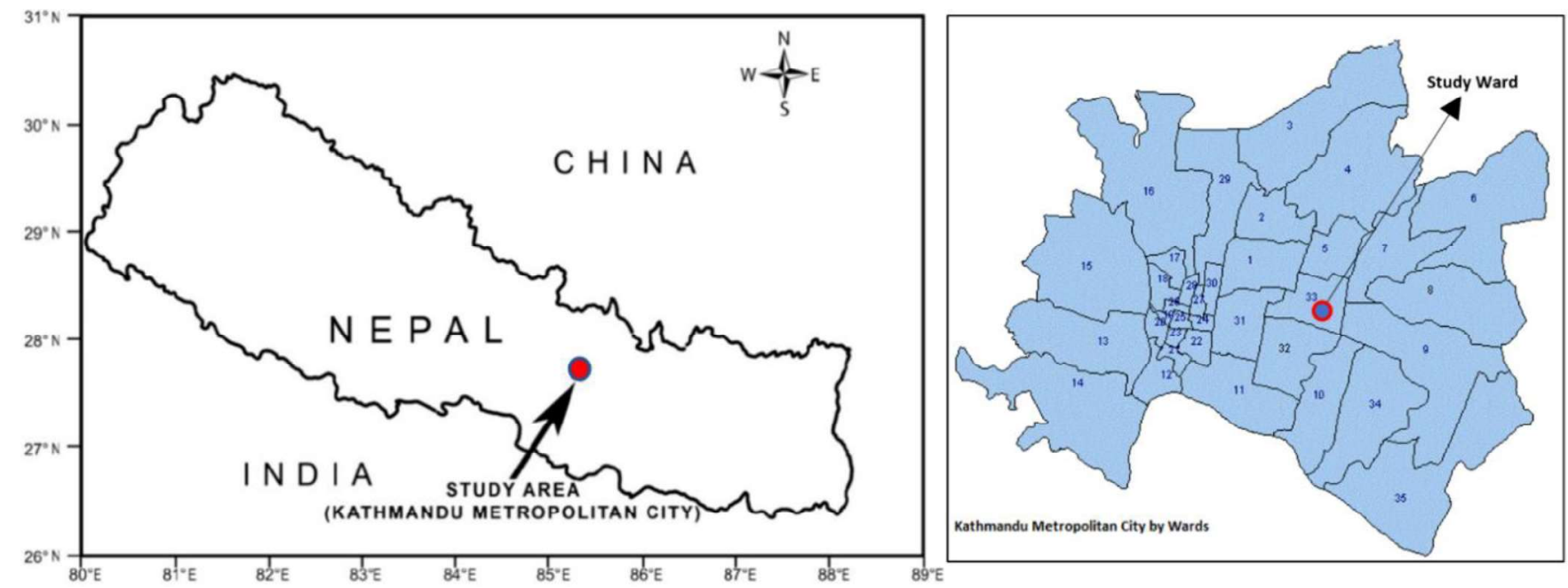

Fig. 1: A map of Nepal showing Kathmandu District (L) and Study Ward-33 (R).

[Source: Adapted from Chhetri \& Kayastha (2015), modified]

As of 2011 , there were $1,744,240$ people living in 436,344 households of this ward with majority of male population (CBS, 2011), with significant growth in the number of population $(21,597)$ as well as household $(5,064)$ compared to the data of 2001 population census of Nepal. With $35 \%$ of the total population of the study ward, Brahmins constituted top ranking followed by Newar (33\%), who are the historical inhabitants of the Kathmandu Valley. Like many other wards of Kathmandu, Ward Number 33 represents a community of diverse ethnic groups who involve in different works and occupations for their livelihoods. Data in this study were collected from primary as well as secondary sources. Based on the data provided by a ward secretary at the time of fieldwork of this study, there were 476 elderly people in this ward. Likewise, secondary data were obtained from government documents, books, journal articles, research reports, and internet sources. Purposive and snowball sampling techniques were used to select survey participants. Profiles of the elderlies maintained by ward office became instrumental for the researcher in the process of research such as to identify the most appropriate participants as well as to locate their places of residence. The results are presented in descriptive form without using table or graph to keep the paper succinct.

\section{Results}

In this section, implications of OAA are reported, discussed and interpreted under two categories, social and economic. Likewise, analyzed data are presented under different headings and sub-headings in a way to meet the study objectives. Generally, livelihood realties of the elderlies can be explored and understood by making detailed analyses of their demographic characteristics as well as social and economic statuses. That means, it is important to understand how old people are treated in their families and how the expenses required for them are met and by whom. Similarly, the issues associated to health and happiness are vital to understand the social security status of elderlies. It is ostensible that older people need more love, support and care from their other family members. However, the reality about these matters may differ from one family to another. This is to say that while some elderlies may have better situations others may not have. Hence, several but relevant inquiries were made to the participants of this study to capture their perceptions and experiences of the implications of OAA on their social as well as economic statuses. A few informal chats were also made with participants who desired to share their views after surveys, and that information was advantageous to us at the time of data triangulation.

\section{Social Implications of Old Age Allowance}

Largely, social implications of old age allowance are viewed as one of the ways of enhancing elderlies' honor and social status. Implications of OAA regarding its societal vicissitudes, especially in the case of developing nations, have been of growing interest among the researchers of recent decades. The results below reveal key inferences of OAA on social aspects of elderlies of the studied ward through several indicators.

\section{Living with Families}

This indicator shows the distribution pattern of respondents by the status of living with families. The results revealed majority participants living with their family members. Only $8 \%$ of the total participants, $(6 \%)$ male and $(2 \%)$ female reported their separation with families. All other participants had at least one child except one female who expressed the reason for this was due to the loss of her husband at her young age and she never got married thereafter. Every participant realized about the need of old age homes in Nepal. While the majority $(85 \%)$ of participants viewed this need as urgency, the rest had no specific answers. However, all participants accorded that the care and love of family members would be more and better than the services provided at old age homes. 
Many participants censured that old age homes in Nepalwere principally profit oriented rather than committed in providing quality services. Participants were also inquired about the number children they had. Compared to male, female participants reported greater number of children. Key causes to increase the number of children in families were viewed as the lack of knowledge about family planning as well as the advantages of nuclear or small sized family among parents. Also, a common cultural tradition of the Nepalese society that necessitates the role of son in family for the purpose of taking care of parents when they get old as well as performing their funeral rituals must have increased the number of children.

\section{Main Care Givers}

In terms of earning livelihoods and performing physical activities, older people are usually unfit and may need other' help (Subedi, 2004). With ageing, people's physical as well as mental health become pathetic (Richeson \& Shelton, 2006). Hence, participants were asked about the state of their overall health situations as well as information about their care takers. Majority of the participants ( $80 \%$ ) informed that their family members (son/daughter/spouse or daughter/sons-in-law) were the ones to take care of them at own homes. None of the participants was found to have stayed in paid or old age homes. However, three participants ( 1 male and 2 females) informed that they lived within the temple premises and survived by eating the food offered to them by pilgrims. Therefore, these participants had no one to take care of them. One of the male participants reported that one of his close relatives was providing him with food and accommodation since last several years. Similarly, other two male participants mentioned that they lived on their own and had no care givers. It is revealed from the study results that compared to male female participants were in better situation in terms of receiving care. Likewise, regarding to the questions about availability of utilities for elderlies at home, while $85 \%$ of the participants reported that they had easy access to drinking water, electricity, telephone, radio, television, and so on, the remaining (15\%) were deprived of these facilities.

\section{Accommodation}

Participants were inquired about their housing conditions. Most of the participants who lived with their families informed that they shared their bedrooms either with spouses and or grandchildren. Furthermore, they reported that their rooms were used as guest rooms when required. However, these participants seemed happy with these adjustments and had no complaints. However, a few (30\%) participants mentioned that due to the lack of heating system inside their bedrooms, winter months were bit difficult. Likewise, $60 \%$ of the participants reported that bathrooms and toilets were not closer to their bedroom, so it was difficult for them use toilets at night.

\section{Food Intake and Sufficiency}

Fresh foods and balanced diet are important for good health. In this context, questions related to food production system and ways of managing food deficiency were asked to explore the state of food sufficiency of the participants' households. Most of the participants reported that their households did not have food deficiency. Only $44 \%$ of the participants' households were food deficient and they managed this problem through several ways. Such as, $64 \%$ managed it through wage labor and remittance, $28 \%$ rent, and $8 \%$ other sources. About the question of food deficiency season/period, most of the participants ( $70 \%$ ) reported a maximum of one to three months and remaining 30\% reported five to seven months. Only one female respondent reported food deficiency for the whole year. As most of the elderlies had no income source in the form of pensions, they were required to seek financial support (money) from other members of their families. Hence, the government provided allowances must be of great support to elderlies' as well as elderlies' households.

\section{Help Provided by Participants to Family}

Likewise, we wanted to know about the types of help that our participants provided to their families. Majority of the participants $(85 \%)$ in male category informed that due to age factor and physical weaknesses, they were unable to help their families in a way they really wanted to. Whereas majority of the female participants $(65 \%)$ stated that they helped their families mostly with the kitchen works such as cooking and chopping vegetables. More than half of the male participants $(55 \%)$ mentioned that they used to help their children and grandchildren in making important decisions over family matters. All male participants said that they helped their families by buying vegetables from the local shops, taking care of their grandchildren and also by feeding them sometimes. Only one female participant said that she used to support her family by giving some of the money from her allowances. Another female participant mentioned that she used to give money to her children and grandchildren when her other children sent money from abroad. Only $24 \%$ of the female participants said that they used to provide suggestions to their family members when important decisions were to be made on household matters.

\section{Feelings of Ageing}

Questions seeking information about socio-psychological feelings of the elderlies such as; sources of happiness and causes of sorrow, wants, desires, relationships and so on were also asked to participants. One of the sentimental questions was about age, to know from when people actually begin to feel older. Most of the male (80\%) and female (92\%) participants viewed a person begins to feel ageing between the age of 50 to 59 years. However, $10 \%$ male and $8 \%$ female participants stated that this stage occurs between $60-69$ years. 
Likewise, $10 \%$ of the male participants reported that people start to feel elderly when they cross 45 years of their age.

\section{Main Social Security Concern}

Usually, when people get older, they begin to wander about several things and situations like, what would happen to them in the future, who would take care of them, whether their health condition would remain good or bad, whether their family members would love them or they may have to leave the house because of abhorrence from family, and keep on worrying about these all. During surveys and informal chats, questions regarding these socio-physiological issues were asked to understand respondents' feelings. Mixed responses were found among the male and female participants. While most of the male participants $(90 \%)$ thought money or financial security as the most important thing for any induvial at old age, this to the majority of female participants $(85 \%)$ was worry of their family members regarding their progress and wellbeing. This specifies that the female participants were less worried about themselves and more worried about their children and grandchildren. About the state of relationships between elderlies and their other family members, $38 \%$ said that it was better than before. However, five male and ten female participants did not realize any change in the state of their relationships with other family members. Participants who informed about deteriorated relationships consisted $32 \%$ of the total. Due to the advantages of nuclear or small sized family, its popularity has grown not only in the developed but also in developing nations. The practice of adopting nuclear family has been advancing in Nepal as well, and this has had direct impacts on the lives of elderlies. Increasing practices of disintegrating from joint family have created the scarcity of people to look after their elderlies in many families. The majority of participants (85\%) argued that the children of elderly people in Nepal are usually uninterested in living with their parents, and also do not pay them proper respect and honor. Participants were then asked about their sources of happiness. The majority of participants perceived healthy state of their life as the main source of happiness, joy and pleasure. Similarly, family support and financial strength were considered other key factors to keep elderlies satisfied. Likewise, other activities and moments that make elderlies enchanted are the respect/honor from family and society; their involvement in religious and spiritual events; as well as occasions for them to donate and contribute to humanitarian or communal works. On the other hand, reasons to make elderlies distressed and unhappy were the dislikes and negligence shown towards them by their family members, followed by elderlies' own ill health situation, loneliness, financial insecurity, physical disability as well as unsuccess of their family members.

\section{Change in Social Status}

Old age allowance and pensions are regular and significant components of income. Although the amount of elderly allowance in Nepal is still insignificant, with this money its receivers must have been able to minimize some of their financial burdens. Concerning to this issue, the first question asked to participants was about the disbursement of allowances. According to new rules, old age allowances and pensions are not provided to recipients in person or at their residences, Rather, they are paid to their bank accounts. The majority of participants thought that it would be an easy and trouble-free way for them to receive allowances if they could be dispensed at their residences. These participants viewed that paperwork and burden of traveling to and from bank were both difficult for elderlies given their physical weaknesses and illiterate characteristics to accomplish such tasks. Another inquiry was about their prestige in family and society as senior citizen. Majority of theparticipants in both categories (male- $60 \%$ and female- $85 \%$ ) stayed 'neutral' to this question. Compared to female, majority of the male participants felt that being a senior citizen is being somewhat prestigious than before. About the engagement of elderlies in social activities, most of the male participants (65\%) mentioned that they involved in social clubs and religious activities in the local area. Whereas the involvement of female in such activities was found minimal (30\%). Percentage of the participants to visit religious institutes or temples was more in female $(90 \%)$ and less in male $(80 \%)$ category. Key purpose of visiting religious premises were for worshiping and performing hymns in the belief of receiving god's blessings. Elderlies believe that these activities are performed to keep their mental state in peace, confess over committed sins, as well as give continuity to the religious practices, culture and tradition of worshiping deities and following Hinduism. However, all respondents realized about the lack of senior citizen club in their surroundings.

\section{Economic Implications of Old Age Allowance}

The results below reveal economic implications of OAA on elderlies' livelihood in the context of the studied ward. This will demonstrate their income sources and property statuses, management of expenditures required for them in everyday situations, including the state of access and constraints that elderlies might have faced while receiving allowances.

\section{Employment/Income of Elderlies' Households}

Majority of the male participants (72\%) reported that they worked in different sectors in the past comprising (22\%) government, (20\%) private company, and (30\%) agriculture. On the other hand, $84 \%$ of the female participants reported themselves as solely housewives. However, the remaining $16 \%$ female participants informed that they were government retirees. To reveal the status of income source and financial situation of the participants' households, the amount of monthly salary or income of every employed household members were tracked. The majority of respondents in male category $(55 \%)$ and nearly half $(45 \%)$ in female category reported that their families earned at least Nrs. 10,000 per 
month. Of this, $30 \%$ male and $35 \%$ female participants fell under the monthly income of Nrs. (5,000 to 10,000). Likewise, $25 \%$ of the male and $10 \%$ of the female participants informed that the average income of their households was between Nrs. (5,000 and 7,000). Daily wage works, self-employment, and house rent were reported as main sources of income for most of the studied households.

\section{Land Ownership}

Land ownership is a key indicator that signals the authority over the economic life of family. Ownership of land makes a household economically strong as well as helps in signifying socioeconomic statuses of the household members. The study results showed $76 \%$ of the male $56 \%$ of the female participants had land ownerships. The majority of participants had crop fields in the Terai belt of Nepal, from where they used to bring harvested crops, such as rice and lentils. Most of the participants thought that this transaction was very instrumental for them in minimizing the cost of grocery in Kathmandu.

\section{Additional Sources of Income}

Obviously, strong financial situations can help manage expenses without burden. For example, to undergo a major health treatment or pay big debt, small amount of money is insufficient. Regarding this issue, participants were asked about how they would manage if they required a big sum of money. $60 \%$ of the male and $72 \%$ of the female participants mentioned that their sons/daughters and son/daughters-inlaws were the ones to manage their financial needs. However, about $35 \%$ of the male as well as female participants stated that their grandchildren were the ones to provide them with big sum of money at the time of need such as for health checkups, buying medicines and going on lengthy pilgrimages. This shows that not only the grandparents take care of their grandchildren, but also the grandchildren do love their grandparents physically and financially.

\section{Regular Expenditures on Medication}

Participants were inquired about the sources and amount of expenses required for buying their regular medicines. The majority of participants in both categories (male- 60\% andfemale-75\%) reported that they required approximately Nrs. 2,000 to 4,000 per month to buy their regular medicines. however, they were unsure about the amount of money that might be required for their health checkup throughout a year. $20 \%$ of the male and $25 \%$ of the female participants informed that they were not under regular medication. The participants who said that they might need about Nrs. 20,000 for their health treatment in a year was found $8 \%$, but only in the female category. This indicated that compared to male, the health condition of female participants was poor and were thus in need of more money for buying medicines and undergoing frequent health checkups. In Nepal, there is a government provision of providing annual financial assistance of about Nrs. 4,000 to an elderly for the health checkups. Participants were thus asked whether they were aware of this provision. $80 \%$ of the male and $70 \%$ of the female participants informed that they were unknown about this advantage. However, two male participants reported that they received this kind of financial assistance in the past.

\section{Awareness of Financial Support for Special Treatment To} expand on the earlier question, participants were again asked whether they were aware of the government provided special financial support of Nrs. 5,000 for elderlies above 75 years to undergo their major treatment such as (kidney, heart, uterine prolapsed and cancer). Almost all participants in both categories were unknown about this provision. Only one male participant said that he was aware of this facility. This means that the concerned authorities were unsuccessful in publicizing the information about this government provided financial support to elderlies. Due to the lack of information about this provision, many helpless and poor elderlies who might have been benefited from this service are being deprived of it. Some of the easy ways of circulating such information to public would be by posting information on the walls and notice boards of all health centers and public places as well as broadcasting through radios and televisions. However, only two participants, one male and one female mentioned that they had received discounts on health treatment in public hospitals. Similarly, inquiries about the awareness of discounts for elderlies on public transportation and mental health check-up were made to participants to which more than $50 \%$ of the male $90 \%$ of the female participants responded that they had never heard of these provisions.

\section{Constraints in OAA Disbursement and its Utilization}

The money received in the form of elderly allowance or pension are important for elderlies as they can buy goods of their own choices with this money. Also, because of this money they do not have to wait and seek for financial help from others or their family members. Unfortunately, there were three participants who complained that their allowances were collapsed. They reported that the bank did not give their allowances saying that they were not drawn on time. However, these participants were unknown about the rule that if allowances are not received by deadline, they get collapse. Likewise, the participants had mixed answers to the question related to the use of allowances. The majority of participants mentioned that their allowances were used for pocket money and sometimes for purchasing groceries for family. However, the percentage of participants to use allowances as pocket money was higher in male (80\%) and lower in female (40\%) categories. Groceries generally include kitchen items such as; milk, salt, sugar, broom, soap, toothpaste, vegetables and so on. Most of the participants mentioned that they also used to 
give some of their allowance money to their grandchildren. Only $20 \%$ of the total participants reported that they used their allowances to purchase medicines for themselves.

\section{Conclusions}

This study attempted to explore social and economic implications of old age allowance (OAA) in Nepal based on the case study of Ward Number 33 of Kathmandu District. Overall, the implications of OAA were found more on economic compared to social aspects of elderlies. Elderlies rendered that the government's provision of providing elderly allowance in Nepal was praiseworthy. However, the amount of OAA was deemed nominal given the towering prices of goods and services, particularly in Kathmandu. The amount of OAA for elderly male was useful as their pocket money and also in buying everyday household goods such as milk and vegetables for their households. This in the case female participants was just opposite as most of their allowances were used in buying everyday kitchen goods and less for pocket money. However, the recipients of OAA admitted that the role of allowances in enriching their social honor was uninfluential. Key social security concerns of elderlies are related to financial and sociopsychological. It was revealed that the source of elderlies' happiness and sadness depend on the behaviors or attitudes of their family members towards them. Majority of the elderlies are taken care by their family members. Common problems of elderlies regarding accommodation and physical facilities are the lack of easy access to toilet and bathroom and heating system in the bedroom, especially during the winter months and at night. Majority of the elderly people were provided with healthy and enough food with average of three meals per day. Senior citizen clubs were regarded as one of the important avenues to unite elderlies of the same community. These platforms were viewed as the occasions for sharing elderlies' feelings and experiences with each other. Likewise, the activities to keep elderlies get rid of their stresses, loneliness and monotony were told reported regularly performed religious activities at homes and temples. In other words, activities of going to temples for worshiping, preaching, and hymning were the most desired acts of elderlies to keep them happy and satisfied with their life. We do expect that this study identified some of the contemporary problems of and prospects for the elderly people of Nepal concerning to the scope and implications of OAA in the country. Our anticipation is that the future studies concerned with the issues of elderlies, such as ageing, old age allowance, and social security, particularly in the context of Nepal should benefit from this paper as a good source of literature. Also, we believe that the results and recommendations of this study may help several government and non-government organizations, particularly working in the field of geriatric in Nepal for reshaping their existing programs and policies. Finally, this study also provides directions for the future research in the field of social security concerns of Nepal. However, given a smaller size sample for this study, we do not recommend generalization of our findings.

\section{References}

Agrawal A (2016) Disability among the elder population of India: A public health concern. Journal of Medical Society 30:15-9.

Central Bureau of Statistics (CBS) (2011) Statistical Pocket Book of Nepal. Central Bureau of Statistics.

Chalise HN (2006) Demographic situation of population ageing in Nepal. Kathmandu University. Medical Journal 4: 354- 362.

Cossar R (2012) Portraits of Aging Men in Late Medieval Italy. The Gerontologist 52: 553-560.

Donaldson JA, Smith CJ, Balakrishnan B, Kadir MM and Mudaliar S (2015) Elderly population in Singapore: Understanding social, physical and financial needs. Singapore: Lien Centre for Social Innovation.

Geriatric Centre Nepal (GCN) (2010) Status Report on Elderly People $(60+)$ in Nepal on Health, Nutrition and Social Status Focusing on Research Needs. Research Prepared for Gov. of Nepal Ministry of Health and population, Kathmandu.

Ghimire S, Baral BK, Karmacharya I, Callahan K and Mishra SR (2018) Life satisfaction among elderly patients in Nepal: associations with nutritional and mental well-being. Health and quality of life outcomes 16: 118.

Jadhav A (2018) Personal Hygiene Practices of Elderly Among Lambani Community. Laxmi Book Publication: India.

Khanal DR (2013) Social security/social protection in Nepal: situation analysis. ILO Country Office for Nepal.

Mosher S (2017) Population control: Real costs, illusory benefits. Routledge.

Nyanguru AC (2007) Migration and aging: The case of Zimbabwe. Journal of aging \& social policy 19: 57-85.

Parker S, Khatri R, Cook IG and Pant B (2014) Theorizing aging in Nepal: beyond the biomedical model. Canadian Journal of Sociology 39: 231-254.

Pun DP and BP Subedi (2009) A social change and the senior citizen in Nepal: A case study of their socio-spatial exclusion. Research submitted to the Social Inclusion Research Fund Secretariat, SNV, Bakhundole, Nepal.

Regmi RR (1993) Socio Economic and Cultural Aspect of Ageing in Nepal. The Occasional Paper in Sociology and Anthropology 3: $28-46$.

Richeson JA and Shelton JN (2006) A social psychological perspective on the stigmatization of older adults. When I'm 64 : 174-208.

Shrestha HS and Satyal VR (2003) Social security and Pension in Nepal. South Asian Network of Economic Institutes

(SANEI).

Subedi BP (2004) The aged and the marginal: Social geography of older people in Nepal. Himalayan Review 1-18. 
World Health Organization (WHO) (2010) Definition of an older or elderly person. Retrieved from

http://www.who.int/healthinfo/survey/ageingdefnolder/en/in dex.html 